\title{
A hybrid method combining the TD-MoM with the TD-UTD to calculate the transient radiation of thin dipoles in presence of a perfectly conducting screen
}

\section{A. Becker and V. Hansen}

Chair of Electromagnetic Theory, University of Wuppertal, Gaußstr. 20, 42097 Wuppertal, Germany

\begin{abstract}
A time domain Method of Moments algorithm (TD-MoM) is combined with the time domain version of the Uniform Theory of Diffraction (TD-UTD). By use of the TDMoM the fields radiated from thin wire structures positioned in free space are determined. The TD-UTD is applied to calculate the fields scattered by a perfectly conducting screen. Both techniques are hybridized by adding possible reflected and diffracted fields in the TD-MoM algorithm and by using the TD-MoM solution to calculate the fields which impinge on to the screen. To show the accuracy of the developed hybrid method, numerical results are compared to results obtained by established frequency domain numerical codes.
\end{abstract}

\section{Introduction}

Any numerical technique has specific advantages and disadvantages: e.g. techniques based on the MoM enable the computation of currents flowing on antennas and scatterers with high accuracy but they require a relatively large amount of memory and CPU-time. Asymptotic methods like the UTD are applicable for the calculation of fields scattered by large and simple bodies with only a small amount of memory and CPU-time but they cannot be used to determine the radiation properties of antennas. For many years it has been state of the art to hybridize numerical techniques in order to keep the advantages of the individual methods and to compensate their disadvantages. In the frequency domain there have been many developed hybrid methods, e.g. combination of the UTD with the MoM and the Finite Element Method (Alaydrus et al., 2002). In contrast to the frequency domain relatively few time domain hybrid methods have been developed until now and there is no equivalent to the long established frequency domain UTD and MoM hybrid methods.

Correspondence to: A. Becker

(abecker@uni-wuppertal.de)
Therefore, we present a hybrid method combing both techniques in the time domain.

In this hybrid method the TD-MoM is applied to calculate the fields radiated by thin wire structures in free space. The scattering of these fields is described with the time domain version of the UTD and fields which are scattered back to the antenna are taken into account in the TD-MoM solution procedure. By this full hybridization of both techniques is achieved and the influence of the surroundings both on the currents flowing on the wire structures and on the fields in the observation points is taken into account.

We present several examples to show the accuracy and the efficiency of the proposed hybrid method and it is shown that the TD-MoM can be faster than its frequency domain counterpart when applied to broadband problems.

\section{TD-MoM}

In this paper the TD-MoM is applied to structures involving thin wires with circular cross-sections. Therefore, several assumptions are used which are discussed in detail in the literature (e.g. Gómez et. al., 1992). The most important are that the tangential electric field on the surface of the wires is zero and that the current distribution can be substituted by equivalent currents along the axes of the wires. In the following we shortly repeat the well known MoM-procedure which can be applied also to patch-antennas by using the corresponding spatial basis functions. The electric fields radiated by currents flowing on antennas and scatterers are calculated by using a time domain electric field integral equation (TD-EFIE):

$$
\begin{aligned}
\boldsymbol{E}(\boldsymbol{r}, t)= & \nabla \frac{1}{4 \pi \varepsilon} \int \frac{\int_{0}^{t-\frac{\left|\boldsymbol{r}-\boldsymbol{r}^{\prime}\right|}{c}} \nabla \boldsymbol{J}\left(\boldsymbol{r}^{\prime}, t-\frac{\left|\boldsymbol{r}-\boldsymbol{r}^{\prime}\right|}{c}\right) d t}{\left|\boldsymbol{r}-\boldsymbol{r}^{\prime}\right|} d a^{\prime} \\
& -\frac{\partial}{\partial t} \frac{\mu}{4 \pi} \int \frac{\boldsymbol{J}\left(\boldsymbol{r}^{\prime}, t-\frac{\left|\boldsymbol{r}-\boldsymbol{r}^{\prime}\right|}{c}\right)}{\left|\boldsymbol{r}-\boldsymbol{r}^{\prime}\right|} d a^{\prime} .
\end{aligned}
$$


The spatial dependency of $\boldsymbol{J}\left(\boldsymbol{r}^{\prime}, t\right)$ is approximated by a sum of spatial basis functions multiplied with unknown coefficients:

$\boldsymbol{J}\left(\boldsymbol{r}^{\prime}, t\right)=\sum_{i=1}^{N_{s}} J_{i}(t) \boldsymbol{\beta}_{i}\left(\boldsymbol{r}^{\prime}\right)$,

thus

$\nabla \boldsymbol{J}\left(\boldsymbol{r}^{\prime}, t\right)=\nabla \sum_{i=1}^{N_{s}} J_{i}(t) \boldsymbol{\beta}_{i}\left(\boldsymbol{r}^{\prime}\right)=\sum_{i=1}^{N_{s}} J_{i}(t) \gamma_{i}\left(\boldsymbol{r}^{\prime}\right)$.

Substituting the current distribution in Eq. (1) by Eq. (2) and by using Eq. (3) one receives:

$$
\begin{aligned}
\boldsymbol{E}(\boldsymbol{r}, t)= & \sum_{i=1}^{N_{s}} \int_{A_{i}}\left[\nabla \frac{1}{4 \pi \varepsilon} \frac{\int_{0}^{t-\frac{\left|\boldsymbol{r}-\boldsymbol{r}^{\prime}\right|}{c}} J_{i}\left(t-\frac{\left|\boldsymbol{r}-\boldsymbol{r}^{\prime}\right|}{c}\right) \gamma_{i}\left(\boldsymbol{r}^{\prime}\right) d t}{\left|\boldsymbol{r}-\boldsymbol{r}^{\prime}\right|}\right. \\
& \left.-\frac{\partial}{\partial t} \frac{\mu}{4 \pi} \int_{A_{i}} \frac{J_{i}\left(t-\frac{\left|\boldsymbol{r}-\boldsymbol{r}^{\prime}\right|}{c}\right) \boldsymbol{\beta}_{i}\left(\boldsymbol{r}^{\prime}\right)}{\left|\boldsymbol{r}-\boldsymbol{r}^{\prime}\right|}\right] d a^{\prime}
\end{aligned}
$$

Using the additional approximation

$\boldsymbol{J}\left(\boldsymbol{r}^{\prime}, t\right)=\sum_{i=1}^{N_{s}} \sum_{j=1}^{N_{t}} J_{i, j} \boldsymbol{\beta}_{i}\left(\boldsymbol{r}^{\prime}\right) \mathcal{T}_{j}(t)$

and taking the temporal derivate of the electric field yields:

$$
\begin{aligned}
\frac{\partial}{\partial t} \boldsymbol{E}(\boldsymbol{r}, t) & =\sum_{i=1}^{N_{s}} \sum_{j=1}^{N_{t}}\left[\nabla \frac{1}{4 \pi \varepsilon} \int_{A_{i}} \frac{J_{i, j} \gamma_{i}\left(\boldsymbol{r}^{\prime}\right) \mathcal{T}_{s}\left(t-\frac{\left|\boldsymbol{r}-\boldsymbol{r}^{\prime}\right|}{c}\right)}{\left|\boldsymbol{r}-\boldsymbol{r}^{\prime}\right|}\right. \\
& \left.-\frac{\partial^{2}}{\partial t^{2}} \frac{\mu}{4 \pi} \int_{A_{i}} \frac{J_{i, j} \boldsymbol{\beta}_{i}\left(\boldsymbol{r}^{\prime}\right) \mathcal{T}_{s}\left(t-\frac{\left|\boldsymbol{r}-\boldsymbol{r}^{\prime}\right|}{c}\right)}{\left|\boldsymbol{r}-\boldsymbol{r}^{\prime}\right|}\right] d a^{\prime} .
\end{aligned}
$$

A scalar equation is derived by multiplying Eq. (6) with the test function $\boldsymbol{\beta}_{k}$ and integrating the result over the domain $A_{k}$ of the test function:

$$
\int_{A_{k}} \boldsymbol{\beta}_{k}(\boldsymbol{r}) \frac{\partial}{\partial t} \boldsymbol{E}(\boldsymbol{r}, t) d a=-\sum_{i=1}^{N_{s}} \sum_{j=1}^{N_{t}} J_{i, j} A(i, j, k) d a^{\prime} d a,
$$

with

$$
\begin{gathered}
A(i, j, k)=\int_{A_{k}} \int_{A_{i}} \frac{1}{4 \pi \varepsilon} \frac{\gamma_{i}\left(\boldsymbol{r}^{\prime}\right) \gamma_{k}(\boldsymbol{r}) \tau_{j}\left(t-\frac{\left|\boldsymbol{r}-\boldsymbol{r}^{\prime}\right|}{c}\right)}{\left|\boldsymbol{r}-\boldsymbol{r}^{\prime}\right|} d a^{\prime} d a \\
+\int_{A_{k}} \int_{A_{i}} \frac{\partial^{2}}{\partial t^{2}} \frac{\mu}{4 \pi} \frac{\boldsymbol{\beta}_{k}(\boldsymbol{r}) \boldsymbol{\beta}_{i}\left(\boldsymbol{r}^{\prime}\right) \tau_{j}\left(t-\frac{\left|\boldsymbol{r}-\boldsymbol{r}^{\prime}\right|}{c}\right)}{\left|\boldsymbol{r}-\boldsymbol{r}^{\prime}\right|} d a^{\prime} d a .
\end{gathered}
$$

It is now assumed that all coefficients $J_{i, j}$ are known for $j<n$ and that Eq. (7) is specialized for $t=n \triangle T$, where $\Delta T$ is the time step size:

$$
\begin{aligned}
\sum_{i=1}^{N_{s}} J_{i, n} A(i, n, k)= & -\int_{A_{k}} \boldsymbol{\beta}_{k}(\boldsymbol{r}) \frac{\partial}{\partial t} \boldsymbol{E}(\boldsymbol{r}, n \triangle T) d a \\
& -\sum_{i=1}^{N_{s}} \sum_{j=1}^{n-1} J_{i, j} A(i, j, k) .
\end{aligned}
$$

All known preceding coefficients (i.e. coefficients corresponding to time steps earlier than the actual time step $n$ ) are written on the right side and all unknown actual coefficients (i.e. currents allocated to the actual time step) are written on the left side of Eq. (8). By fulfilling Eq. (8) at the domains $A_{k}$ of all test functions $\boldsymbol{\beta}_{k}$, the currents at the actual time step can be calculated by solving the resulting system of linear equations. In the next time step this system of linear equations is built and solved once again. Incoming fields (e.g. produced by a scatterer) and voltage-sources on the wires can be taken into account by adding them to the fields produced by the old and thus known currents.

\section{TD-UTD}

In this chapter a short overview of the theoretical background of the time domain UTD and of its application to the diffraction of transient fields by a perfectly conducting screen is given.

\subsection{Geometrical optical fields}

The well known geometrical optical description of a wave in the frequency domain is given by the field $\boldsymbol{E}_{0}$ in the reference point multiplied with the divergence factor, a potential phase jump and the phase factor $e^{-j k s}$.

$\boldsymbol{E}(s, \omega)=\boldsymbol{E}_{0}(\omega) \sqrt{\frac{\rho_{1} \rho_{2}}{\left(\rho_{1}+s\right)\left(\rho_{2}+s\right)}} j^{n} e^{-j k s}$,

where $\mathrm{n}$ is the number of caustics the wave crosses after having passed the reference point, $s$ is the distance of the observation point from the reference point and $\rho_{1}$ and $\rho_{1}$ are the principal radii of curvature for the corresponding waveform at the reference point. When applying the inverse Fourier transform separate analysis is necessary according to the number of caustics passed. To avoid this inconvenience one can use the analytic time function representation of the electric field:

$\stackrel{+}{\boldsymbol{E}}(s, t)=\stackrel{+}{\boldsymbol{E}}_{0}\left(t-\frac{s}{c}\right) \sqrt{\frac{\rho_{1} \rho_{2}}{\left(\rho_{1}+s\right)\left(\rho_{2}+s\right)}} j^{n}$.

The real time function representation can be found by taking the real part of the analytic signal representation:

$\boldsymbol{E}(s, t)=\operatorname{Re}\{\stackrel{+}{\boldsymbol{E}}(s, t)\}$.

3.2 UTD description of the field radiated by a source in presence of a perfectly conducting wedge

The field radiated by a source in presence of a perfectly conducting wedge can be approximated as the sum of the direct, the reflected and the diffracted field:

$\stackrel{+}{\boldsymbol{E}}(s, t)=\stackrel{+}{\boldsymbol{E}}_{L O S}(s, t) U_{i}+\stackrel{+}{\boldsymbol{E}}_{R}(s, t) U_{R}+\stackrel{+}{\boldsymbol{E}}_{D}(s, t)$,

with $U_{i}=1$ if line of sight (LOS) is given between source and observation point and $U_{i}=0$ if no direct field reaches the observation point. $U_{R}$ is one, if a reflected field reaches 
the observation point and it is zero, otherwise. Both $U_{i}$ and $U_{R}$, as well as possible reflection and diffraction points are found by using a ray-tracing algorithm. After performing ray-tracing the reflected field is found by multiplying the field $\mathbf{E}_{R_{0}}^{+}$in the reflection point with the dyadic reflection coefficient, the divergence factor and a potential phase jump $j^{n_{r}}$ :

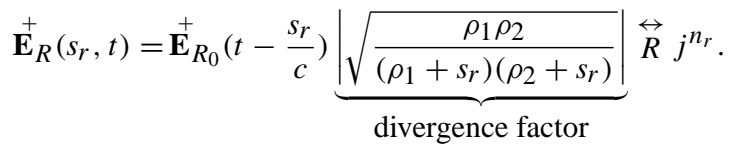

Due to the frequency dependence of the diffraction coefficient the diffracted field is found by convolution of the field ${\stackrel{+}{\mathbf{E}_{D_{0}}}}_{\text {in }}$ the diffraction point with the diffraction coefficient. The result is multiplied with the divergence factor and a potential phase jump:

${\stackrel{+}{\mathbf{E}_{D}}}_{\left(s_{d}, t\right)}=\stackrel{+}{\mathbf{E}}_{D_{0}}\left(t-\frac{s_{d}}{c}\right) * \overleftrightarrow{d}\left(t-\frac{s+s_{d}}{c}\right) A_{d}\left(s_{d}\right) j^{n_{d}}$

The dyadic diffraction coefficient $\stackrel{\leftrightarrow}{d}\left(t-\frac{s+s_{d}}{c}\right)$ is an analytic function in time which is derived by taking the inverse analytic Laplace Transform of the FD-diffraction coefficient (Rousseau and Pathak, 1995). The dyadic diffraction coefficient is given by:

$$
\overleftrightarrow{d}(t)=-\boldsymbol{\beta}^{\prime} \boldsymbol{\beta}{\stackrel{+}{d_{s}}}_{(}(t)-\boldsymbol{\phi}^{\prime} \boldsymbol{\phi} \stackrel{+}{d_{h}}(t)
$$

For details it is referred to the literature (e.g. Kouyoumjian and Pathak, 1974).

3.3 Edge diffraction of a spherical wave by a flat PEC screen with straight edge

The scalar diffraction coefficients for a perfectly conducting flat screen (see Fig. 1) are given by:

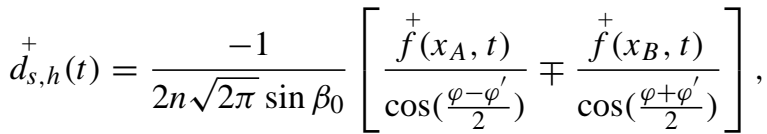

with

$x_{A}=2 L \cos ^{2}\left(\frac{\varphi-\varphi^{\prime}}{2}\right)$

and

$x_{B}=2 L \cos ^{2}\left(\frac{\varphi+\varphi^{\prime}}{2}\right)$.

The analytic function $\stackrel{+}{f}\left(x_{m}, t\right)$ reads

$\stackrel{+}{f}\left(x_{m}, t\right)=\frac{\sqrt{x_{m} / \pi}}{\sqrt{-j t}\left(\sqrt{-j t}+\sqrt{j x_{m} / c}\right)}$.

If the incoming field passes no caustic it is more convenient to work with the real time function

$f\left(x_{m}, t\right)=\operatorname{Re}\left\{\stackrel{+}{f}\left(x_{m}, t\right)\right\}=\frac{x_{m} / \sqrt{\pi c}}{\sqrt{t}\left(t+x_{m} / c\right)} u(t)$.

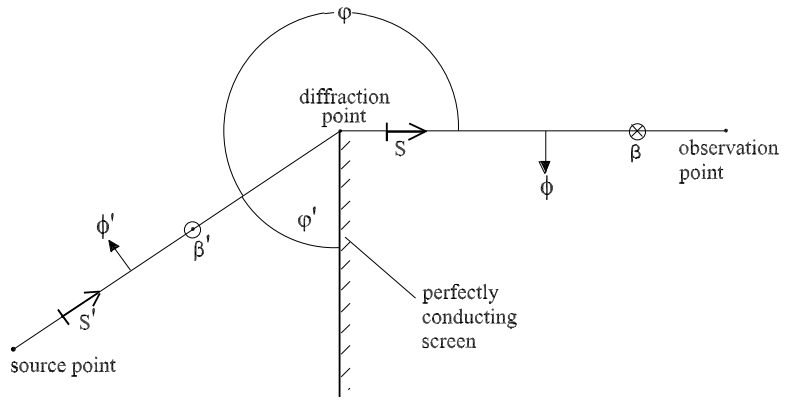

Fig. 1. Diffraction by a perfectly conducting flat screen.

This impulse response becomes singular for $t=0$ and steepens with decreasing $x_{m}$. Using Eqs. (16), (17), (18) and (20) leads to:

$$
\begin{aligned}
d_{s, h}(t)= & \frac{-1}{2 n \sqrt{2 \pi} \sin \beta_{0}}\left[\frac{2 L \cos \left(\frac{\varphi-\varphi^{\prime}}{2}\right) / \sqrt{\pi c}}{\sqrt{t}\left(t+x_{A} / c\right)} u(t)\right. \\
& \left.\mp \frac{2 L\left(\frac{\varphi+\varphi^{\prime}}{2}\right) / \sqrt{\pi c}}{\sqrt{t}\left(t+x_{B} / c\right)} u(t)\right] \\
= & \frac{-1}{2 n \sqrt{2 \pi} \sin \beta_{0}}[A(t) \mp B(t)] .
\end{aligned}
$$

At the radiation or shadow boundary either $A(t)$ or $B(t)$ must be impulsive in time coresponding to the FD-UTD where one of both becomes frequency independent. Consequently, for observation points very close to a shadow or reflection boundary numerical integration is not suited to perform the convolution in Eq. (14). With

$$
\int_{0}^{\infty} \frac{1}{\sqrt{t}\left(t+\frac{x_{m}}{c}\right)} d t=\pi \sqrt{\frac{c}{x_{m}}}
$$

one can derive for the shadow boundary:

$$
\begin{aligned}
A(t) & =\delta(t) \frac{2 L \cos \left(\frac{\phi-\phi^{\prime}}{2}\right)}{\sqrt{\pi c}} \pi \sqrt{\frac{c}{2 L \cos ^{2}\left(\frac{\phi-\phi^{\prime}}{2}\right)}} \\
& =\sqrt{2 L \pi} \delta(t) .
\end{aligned}
$$

For the reflection boundary follows:

$B(t)=\sqrt{2 L \pi} \delta(t)$.

In the proposed hybrid method the fields emitted by the antenna currents are modelled as spherical waves and thus $L$ becomes

$L=\frac{s s^{\prime}}{s+s^{\prime}} \sin ^{2} \beta_{0}$. 


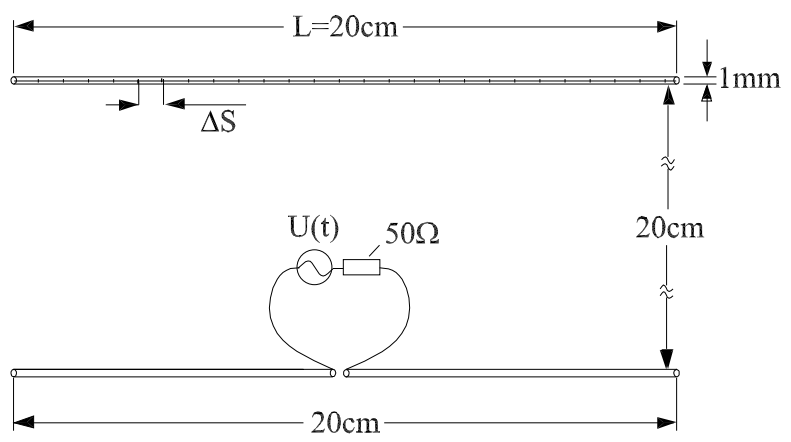

Fig. 2. Geometry of dipole array.

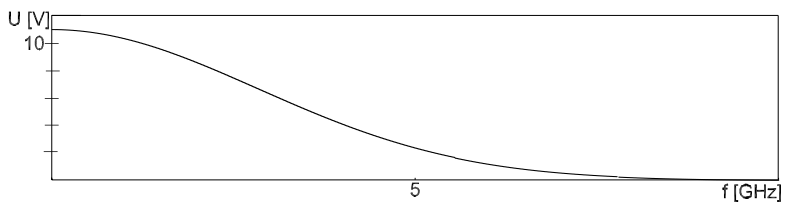

Fig. 3. Spectrum of the applied voltage.

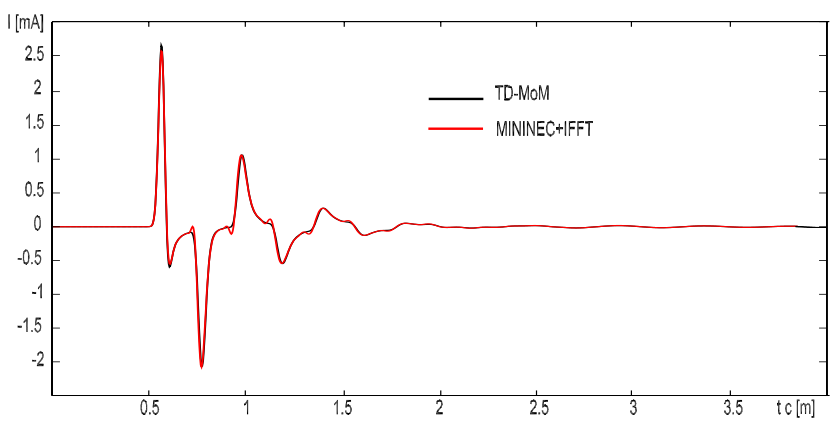

Fig. 4. Temporal behaviour of the source current.

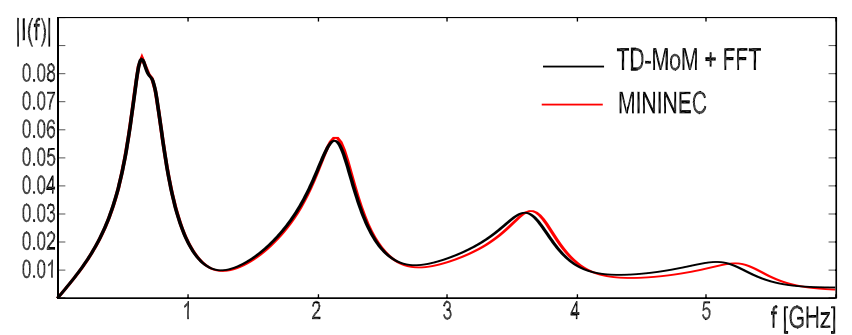

Fig. 5. Spectrum of the source current.

\section{Hybrid Method}

Hybridization of the TD-UTD and the TD-MoM leads to a method which can consider the full influence of the surroundings on the antenna currents and on the fields emitted by these currents. By using ray tracing and by adding possible reflected and diffracted fields to Eq. (8) one receives:

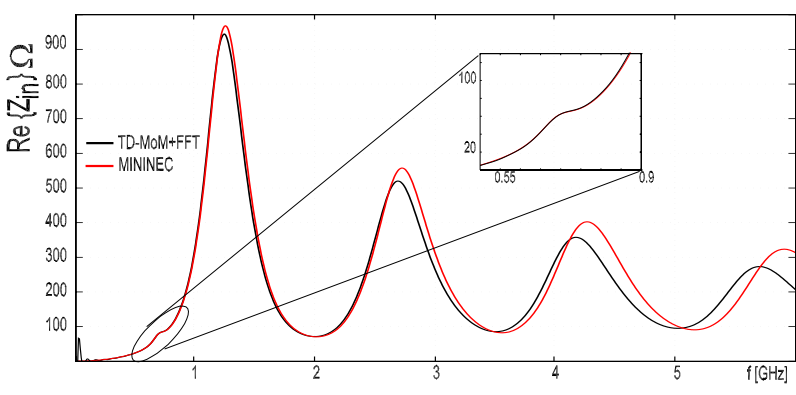

Fig. 6. Real part of the input impedance.

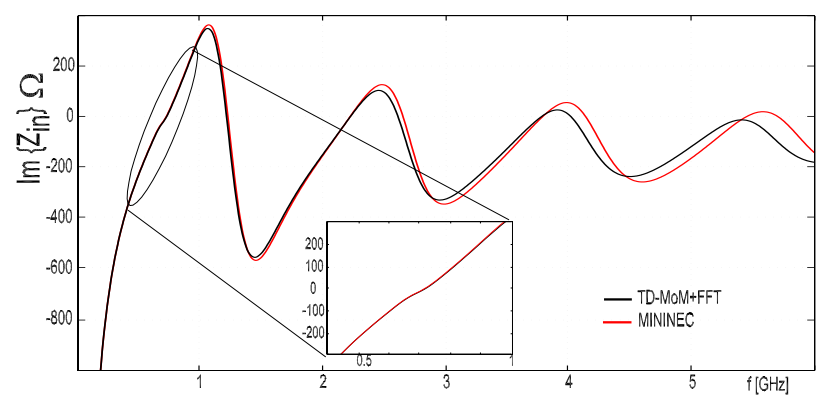

Fig. 7. Imaginary part of input impedance

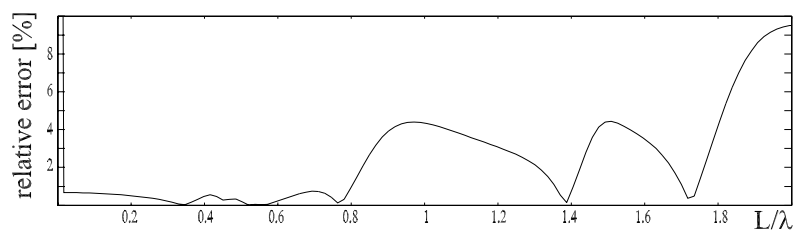

Fig. 8. Relative error of the magnitude of the input impedance as a function of the relative length of the dipole

$$
\begin{aligned}
& \sum_{j=1}^{N_{s}} J_{i, n} U_{L O S, i, k} A(i, n, k)=-\int_{A_{k}} \boldsymbol{\beta}_{k}(\boldsymbol{r}) \frac{\partial}{\partial t} \boldsymbol{E}(\boldsymbol{r}, n \triangle T) \\
& -\sum_{i=1}^{N_{s}} \sum_{j=1}^{n-1} J_{i, j} U_{L O S, i, k} A(i, j, k) \\
& -\left.\int_{A_{k}} \sum_{i=1}^{N_{s}} \sum_{j=1}^{n-1} \boldsymbol{\beta}_{k}(\boldsymbol{r}) \boldsymbol{E}_{D+R}(i, j, \boldsymbol{r})\right|_{t=n \triangle T}
\end{aligned}
$$

$U_{L O S, i, k}=1$ if $\operatorname{LOS}$ is given between the basis function $\boldsymbol{\beta}_{\boldsymbol{i}}$ and the test function $\boldsymbol{\beta}_{k}$ and $U_{L O S, i, k}=0$, otherwise. $\left.\boldsymbol{E}_{D+R}(i, j, \boldsymbol{r})\right|_{t=n \Delta T}$ describes the fields radiated by $J_{i, j}$ which reaches the domain of the test function $\boldsymbol{\beta}_{k}$ (at the time step $n T$ ) after reflection or diffraction. When using Eq. (26) instead of Eq. (8) in the TD-MoM solution process the influence of the surroundings on the antenna currents is taken into account. According to Eq. (12) the fields in the observation points are found by addition of possible direct, reflected and diffracted fields. The direct fields are calculated by using Eq. (1). The reflected and diffracted fields are found by 


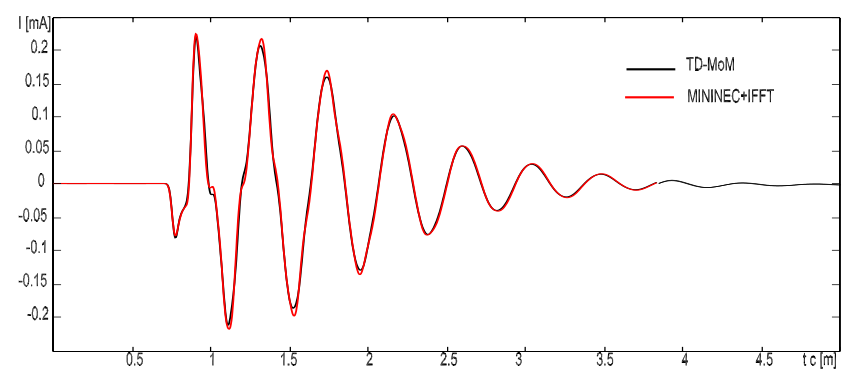

Fig. 9. Temporal behaviour of the center-point current on the second dipole

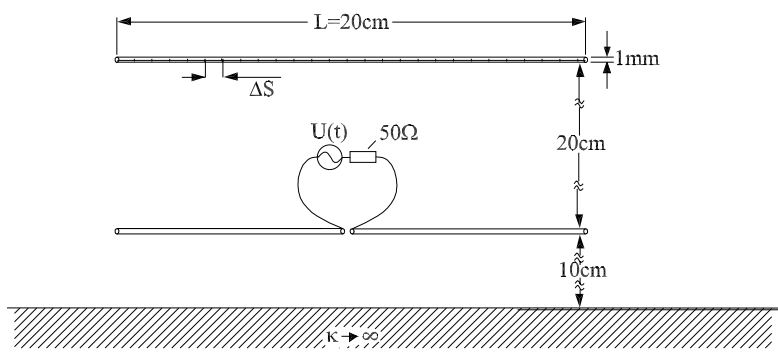

Fig. 10. Geometry of dipole array above perfectly conducting ground.

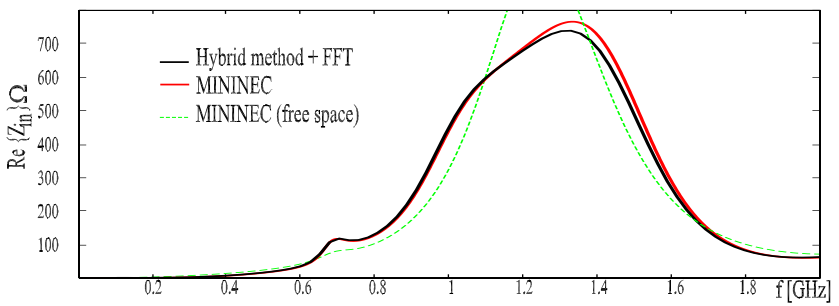

Fig. 11. Real part of the input impedance of the dipole array above perfectly conducting ground.

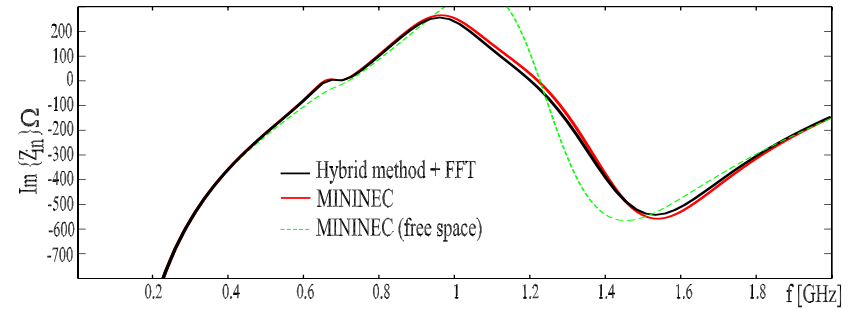

Fig. 12. Imaginary part of the input impedance of the dipole array above perfectly conducting ground.

calculating the fields in the reflection/diffraction points with Eq. (1) and by applying Eq. (13) or Eq. (14), respectively.

\section{Numerical Examples}

In this section the properties of the proposed hybrid method are evaluated by comparing results with results obtained by

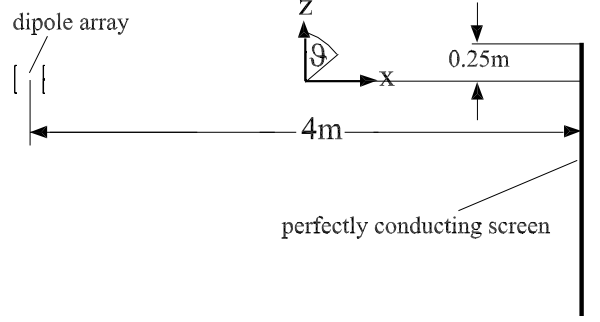

Fig. 13. Geometry of dipole array in front of a perfectly conducting screen.

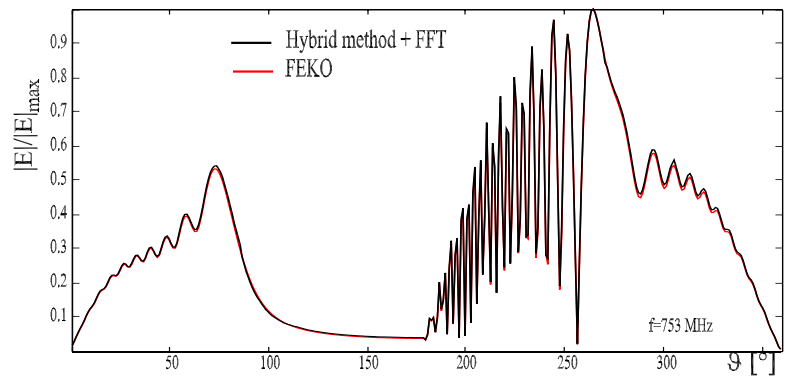

Fig. 14. Normalized far field radiation pattern of the array in front of a perfectly conducting screen $(\mathrm{f}=753 \mathrm{MHz})$.

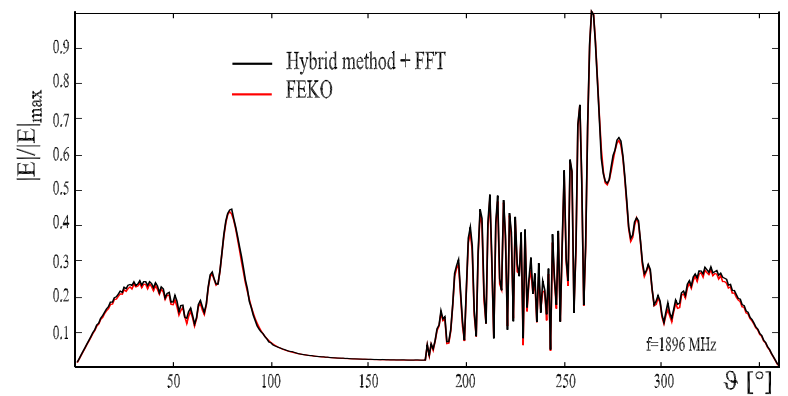

Fig. 15. Normalized far field radiation pattern of the array in front of a perfectly conducting screen $(f=1896 \mathrm{MHz})$.

using long established frequency domain algorithms. For this purpose the dipole array shown in Fig. 2 is studied, positioned in different surroundings. The spatial discretization size $\triangle S$ was chosen to $\triangle S=0.67 \mathrm{~cm}$ for both the hybrid method and the reference methods.

\subsection{Dipole array in free space}

First we consider the array positioned in free space. The applied voltage is a gaussian pulse; its spectrum is shown in Fig. 3. The applied voltage has the following properties:

- Its spectrum is extremely broad.

- Its corresponding wavelength contains components for which the spatial discretization $\Delta S$ is larger than $\frac{\lambda}{10}$.

- It contains a large DC-component. 


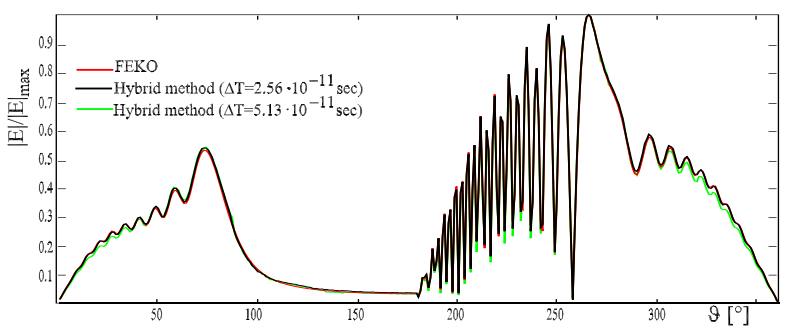

Fig. 16. Normalized far field radiation pattern of the array in front of a perfectly conducting screen $(\mathrm{f}=753 \mathrm{MHz})$ calculated using different time step sizes $\triangle T$.

The time step is chosen according to the Courant limit $\left(\triangle T=1.28126 \cdot 10^{-11} \mathrm{~s}\right)$, although it can be larger without leading to instabilities (see Fig. 16 in Sect. 5.3). In Fig. 4 the resulting source current is compared to the result obtained by using the frequency domain MoM based code MININEC and the inverse FFT. The center-point currents of the second dipole are compared in Fig. 9. The results agree very well. In Fig. 5 both source currents are compared in the frequency domain and it becomes obvious that both agree best at lower frequencies. The calculation time of our time domain MoM was around 8 seconds (3000 time steps, which equals 11.5 light meter) compared to around $32 \mathrm{~s}$ for MININEC (on the same computer, 400 frequency steps, same spatial discretization). In Figs. 6 and 7 the real and the imaginary part of the input impedance are shown, respectively. Around $750 \mathrm{MHz}$ one can see the influence of the second dipole on the input impedance of the first one. For frequencies up to around $1.2 \mathrm{GHz}$ the relative error of the magnitude of the input impedance is smaller than 2\% (see Fig. 8). For higher frequencies the results of the TD-MoM and the FD-MoM diverge more; the TD-MoM shows some kind of dispersion effect: For high frequencies the resonance frequencies of the antenna are lower than obtained by using MININEC.

\subsection{Dipole array above perfectly conducting ground}

As a second example the input impedance of the same array $10 \mathrm{~cm}$ above perfectly conducting ground (see Fig. 10) is investigated. For excitation the same pulse as in Sect. 5.1. was used. The result is compared to the input impedance of the same array calculated with MININEC (Figs. 11 and 12). Additionally, the input impedances of the same array in free space are plotted to show the influence of ground. The results agree well and the strong influence of the ground plane on the input impedance around $750 \mathrm{MHz}$ is taken into account very well.

\subsection{Dipole array in front of a perfectly conducting screen}

The last example is the same array, excited with the same impulse but this time in front of a perfectly conducting screen (Fig. 13). The normalized radiation patterns are compared to the results obtained by using the frequency domain MoM UTD based hybrid method FEKO. As one can see in Figs. 14 and 15 the results agree very well. In Fig. 16 the radiation pattern calculated using different time step sizes is shown for $f=653 \mathrm{MHz}$. Obviously, in the application of the proposed hybrid method it is possible to use much larger time steps than given by the Courant limit.

\section{Conclusions}

The hybrid method presented in this paper combines a time domain Method of Moments algorithm with the time domain version of the Uniform Theory of Diffraction. Several examples were presented and it was shown that our hybrid method can be applied for calculating the transient radiation properties of antennas in complex surroundings. Despite of using extremely broadband excitation pulses with strong DC-component the radiation patterns and input impedances agree very well to long established frequency domain codes. The proposed method can easily be extended to be applied to patch antennas and more complicated UTD-bodies.

\section{References}

Alaydrus, M., Hansen, V., and Eibert, T. F.: Hybrid ${ }^{(2)}$ : Combining the three-dimensional hybrid finite element-boundary integral technique for planar multilayered media with the uniform geometrical theory of diffraction. IEEE Transactions on Antennas and Propagation, vol. 50, no. 1, 67-74, 2002.

Gómez, R., Martin, A., and Rubio Bretones, A.: Time-Domain Integral Equation Methods For Transient Analysis, IEEE AP Magazine, vol. 34, No. 3, 15-23 June 1992.

Rousseau, P. R. and Pathak, P. H.: Time domain Geometrical Theory of Diffraction for a Curved Wedge. IEEE Trans. AP, Vol. 43, no. 12, 1375-1385, Dec. 1995.

Kouyoumjian, R. G. and Pathak, P. H.: A Uniform Geometrical Theory of Diffraction for an Edge in a Perfectly Conducting Surface. Proc. IEEE, Vol. 62, 1448-146, Nov. 1974. 